\title{
One case of papillary thyroid carcinoma complicated with epidermal follicular carcinoma was followed up for four years
}

Wei Yan, Qing-Jun Gao, Rong-Jun Gao, Yan Zhou, Wei Zhang, Ying-Peng, Wen-Li Tang, Xu-Dai, Hui Ye

Department of Thyroid Surgery, Affiliated Hospital of Guizhou Medical University, China

Submitted: 10 December 2021; Accepted: 4 February 2022

Online publication: 24 February 2022

Arch Med Sci 2022; 18 (2): 559-563

DOI: https://doi.org/10.5114/aoms/146426

Copyright $@ 2022$ Termedia \& Banach

Thyroid cancer is a common endocrine malignant tumour. In recent years, the incidence of thyroid cancer has been on the rise [1]. Differentiated thyroid carcinoma (DTC) includes papillary thyroid cancer (PTC) and follicular thyroid cancer (FTC). In this paper, a case of papillary thyroid carcinoma with extensively invasive follicular thyroid carcinoma invading the epidermis was followed up for 4 years, and the pathogenesis, treatment and prognosis of this disease were discussed by consulting the relevant literature.

Medical history: The patient is male, aged 70 . Two years ago, it was found that there was a mass on the side of the neck trachea, about $1 \mathrm{~cm}$ $\times 1 \mathrm{~cm}$, and the mass increased progressively. The number increased to four, of which one mass protruded from the neck skin.

Physical examination: The neck was asymmetrical. Four masses were seen in the anterior area of the neck. The size ranged from $1.0 \mathrm{~cm} \times 2.0 \mathrm{~cm}$ to $2.0 \mathrm{~cm} \times 2.0 \mathrm{~cm}$ (Figure $1 \mathrm{~A}$ ). There was no tenderness, poor mobility, or unclear boundaries, and the texture was hard. The relationship with the left thyroid was unknown. The right thyroid had no obvious masses, and no swollen lymph nodes were felt on the bilateral neck and clavicle.

Supplementary examination: Colour Doppler ultrasound (Figures 2 A-D) showed that the larger nodule of the right lobe was located in the lower part, the size was $15.3 \mathrm{~mm} \times 9.6 \mathrm{~mm}$, and part of the border was unclear. A hypoechoic nodule about $4 \mathrm{~mm} \times 3.8 \mathrm{~mm}$ in the middle and lower right lobe had a clear boundary and less regular edges. There were multiple masses in the left part of the neck. The largest one was $27 \mathrm{~mm} \times 21.7 \mathrm{~mm}$, with a clear boundary, equal echo and regular shape. There were abundant strip blood flow signals, and the arterial blood flow spectrum could be detected. Fine needle aspiration cytology (FNAC) inspection was not performed. Neck computed tomography (CT) plain scan + enhanced: There were multiple abnormal enhancement shadows in the front of the thyroid, and lymph node metastasis was to be excluded.

Surgery: During the operation, masses were seen in the anterior part of the neck. The largest was about $2.0 \mathrm{~cm} \times 1.5 \mathrm{~cm}$ and had invaded the anterior cervical skin and subcutaneous anterior cervical muscle layer and extended down to the left thyroid. There was a thyroid bilobar solid mass, homogeneous and tough, without a complete capsule. There were multiple enlarged lymph nodes at the bilateral tracheoesophageal sulcus and right carotid sheath, with a diameter of $0.5-1.5 \mathrm{~cm}$. During the operation, considering that the patient's focus invaded outside the skin,

\author{
Corresponding author: \\ Hui Ye \\ Department of \\ Thyroid Surgery \\ Affiliated Hospital of \\ Guizhou Medical University \\ China \\ E-mail: 1710681307@qq.com
}



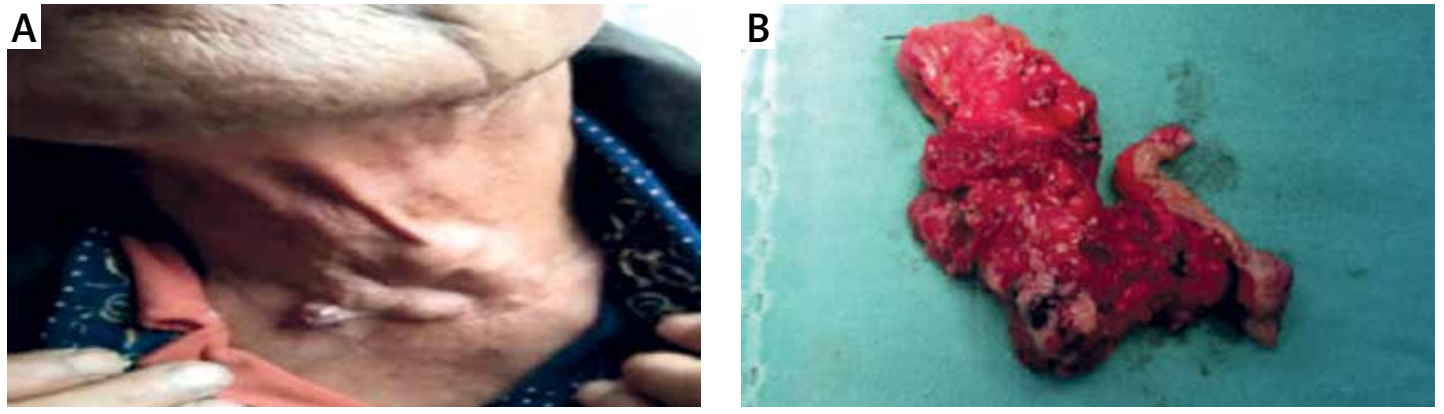

Figure 1. A - Visual examination of the patient's neck: the neck was asymmetrical, 4 masses were seen in the anterior area of the neck, the size was between $1.0 \mathrm{~cm} \times 2.0 \mathrm{~cm}$ and $2.0 \mathrm{~cm} \times 2.0 \mathrm{~cm}$. B - Anterior cervical subcutaneous mass and left thyroid tissue
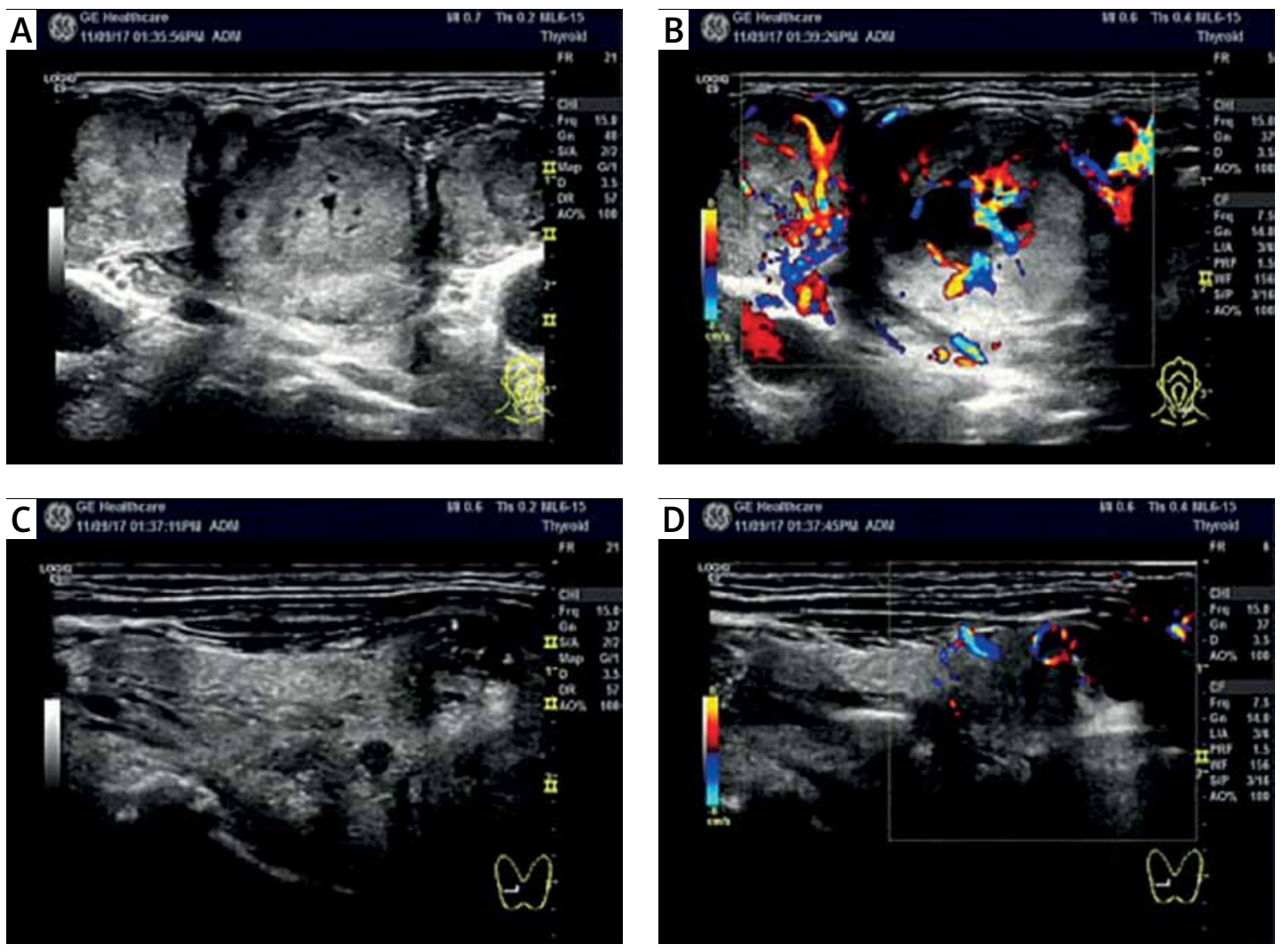

Figure 2. A, B - Patient colour Doppler ultrasound: multiple masses in the left neck, the largest one was $27.0 \times$ $21.7 \mathrm{~mm}$, with clear boundary, equal echo and regular shape, there were abundant strip blood flow signals, and the arterial blood flow spectrum was detected. C, D - Patient colour Doppler ultrasound: the larger nodule of the right lobe was located in the lower part, the size was $15.3 \mathrm{~mm} \times 9.6 \mathrm{~mm}$, part of the border was unclear; a hypoechoic nodule with a size of about $4 \mathrm{~mm} \times 3.8 \mathrm{~mm}$ in the middle and lower right lobe had a clear boundary and less regular edges

and cervical colour Doppler ultrasound and CT suggested cervical lymph node metastasis, it was decided to perform total thyroidectomy, bilateral area $\mathrm{VI}$ and lateral cervical lymph node dissection and anterior cervical subcutaneous tumour resection. Rapid intraoperative pathology: right multifocal papillary thyroid carcinoma (MPTC), two lesions, diameter $0.3 \mathrm{~cm}$ and $1 \mathrm{~cm}$, anterior cervical subcutaneous mass and left thyroid gland (Figure $1 \mathrm{~B}$ ): PTC, diameter $1.5 \mathrm{~cm}$, with follicular tumours that tend to be malignant. Slow postoperative pathology (Figures 3 A-F). Left PTC, maximum di- ameter $1.5 \mathrm{~cm}$, combined FTC (extensive invasion type), maximum diameter $3.5 \mathrm{~cm}$, penetrated the thyroid capsule, subcutaneous tissue to the epidermis, right PTC, two lesions, diameter $0.3 \mathrm{~cm}$ and $1 \mathrm{~cm}$. There was no cancer metastasis in the lymph nodes in the second, third, fourth, and sixth areas of the neck on both sides.

Follow-up and prognosis: After the operation, the patient had temporary hypoparathyroidism and no hoarseness. Two months after the operation, the patient was treated with iodine-131 at a dose of $120 \mathrm{mci}$ and levothyroxine tablets 
$100 \mu \mathrm{g} /$ day were taken. The patient was re-examined 3 months after the operation, and there was no hoarseness, numbness or other complications. The patient's thyroid-stimulating hormone (TSH, reference range: $0.270-4.200 \mathrm{mIU} / \mathrm{l}$ ) was controlled at $<0.1 \mathrm{mIU} / \mathrm{l}$, the preoperative thyroglobulin antibody (TGAB, reference range: 0.00-115.00 U/ml) examination was $20.08 \mathrm{U} / \mathrm{ml}$, postoperative thyroglobulin antibody (TGAB, ref- erence range: $0.00-115.00 \mathrm{U} / \mathrm{ml}$ ) examination was always $<15.00 \mathrm{U} / \mathrm{ml}$, thyroglobulin $(\mathrm{Tg}$, reference range: $3.50-77.00 \mathrm{ng} / \mathrm{ml}$ ) examination was always $<1 \mathrm{ng} / \mathrm{ml}$, and the latest review showed TSH $0.756 \mathrm{mIU} / \mathrm{l}$, free triiodothyronine T3 (FT3, reference range: $3.10-6.80 \mathrm{pmol} / \mathrm{l}) 4.93 \mathrm{pmol} / \mathrm{l}$, and free free thyroxine T4 (FT4, reference range: 12.-22.00 pmol/l) $20.79 \mathrm{pmol} / 1$. The endocrine suppression treatment was satisfactory, and the
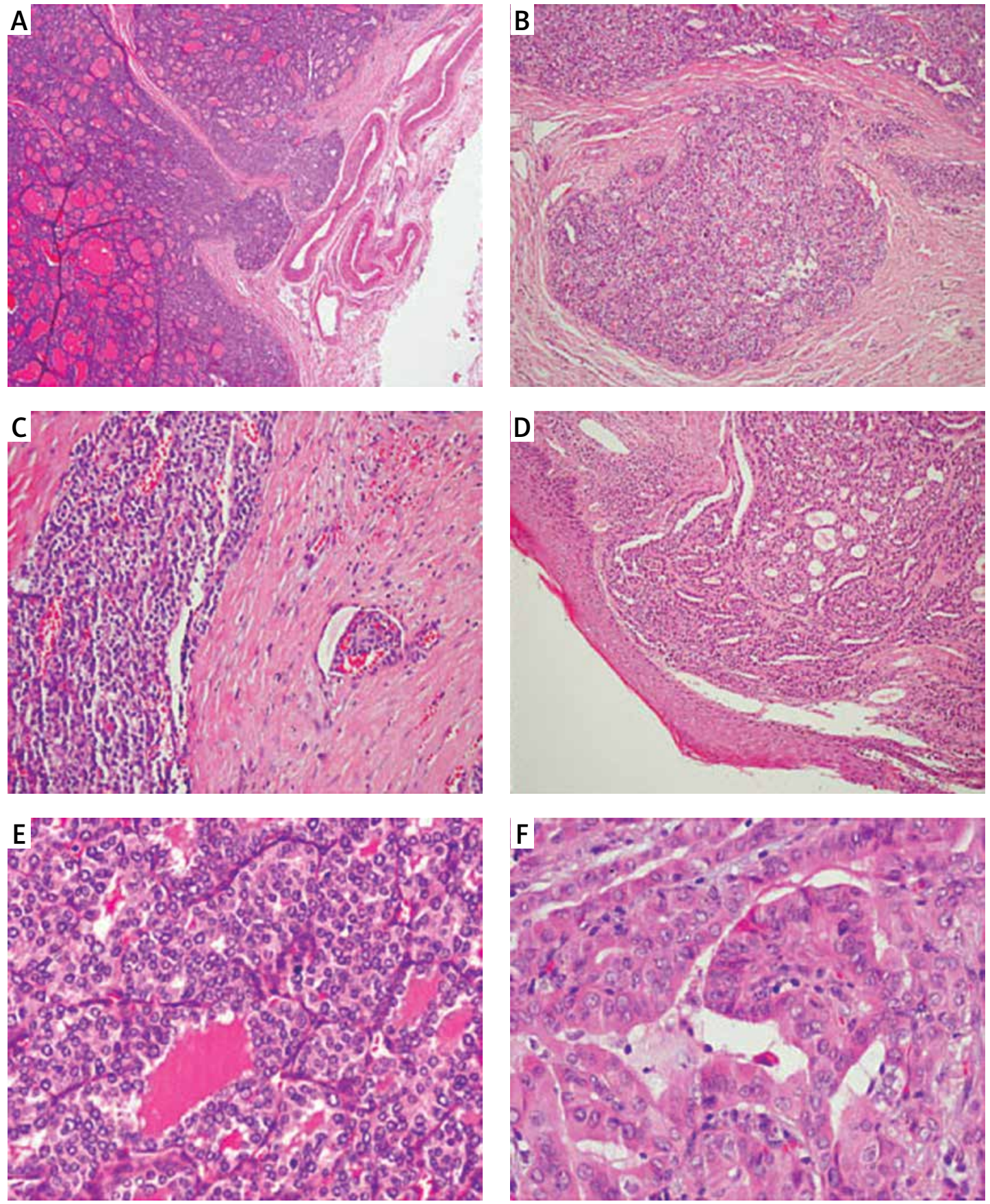

Figure 3. A - Follicular thyroid carcinoma penetrated into the capsule under HE staining at 40× microscope. B - Follicular thyroid carcinoma penetrated through the capsule under HE staining at 200x microscope. C - Follicular thyroid carcinoma vascular invasion under HE staining at 200x microscope. D - Follicular thyroid carcinoma skin invasion under HE staining at $100 \times$ microscope. E - Follicular thyroid carcinoma under HE staining at $400 \times$ microscope. $\mathbf{F}$ - Papillary thyroid carcinoma under HE staining at 400× microscope 
ultrasound examination of the neck showed no abnormalities. The patient has been followed up for 4 years, there is no sign of recurrence or metastasis, and the prognosis is good (Figures 1-3).

The pathogenesis of thyroid cancer is still unclear. Radiation, genetics, excessive or insufficient intake of iodine, and hormones including sex hormones and thyroid-stimulating hormones, are all factors related to the occurrence of thyroid cancer. Its occurrence is a multi-gene and multistep carcinogenesis process [2]. Studies [3] have shown that ret proto-oncogene (RET) rearrangement is more often found in classical PTC, RAS mutation is often found in follicular PTC, and B-Raf proto-oncogene (BRAF) mutation is often found in high cell PTC. In FTC, RAS mutation and peroxisome proliferator activated receptor $\gamma$ (PPARG)-paired box 8 (PAX8) rearrangement are the main genetic abnormalities, and both PTC and FTC have the possibility of phosphatidylinositol-4,5-bisphosphate 3-kinase catalytic subunit $\alpha$ (PIK3CA) amplification and RAS mutation. Other studies [4] have shown that BRAF mutation exists in about $50 \%$ of PTCs, and RAS gene mutation exists in $40-50 \%$ of FTCs. Both BRAF and NRAS proto-oncogene (NRAS) mutations change the mitogen-activated protein kinase 1 (MAPK) pathway of cell proliferation, suggesting that these two tumours may have overlapping molecular pathophysiological changes after inciting genetic aberrations. Both PTC and FTC are follicular cells that originate from the endoderm. They have the same origin and may have overlapping mutation points. Moreover, these two tumours may have overlapping molecular pathophysiological changes after inciting genetic aberrations, so this may be an explanation for the occurrence of PTC and FTC in the primary tumour.

It is also possible that the 'collision theory' [5] causes the occurrence of the disease; that is, the concurrency of PTC and FTC is due to coincidence. Due to the high incidence of PTC, there are patients with FTC who have combined FTC. It has been reported [6] that PTC and FTC can also occur in the thyroid at the same time, but their distant metastasis may not be related. In 1 case, there was PTC in the left lobe of the thyroid and papillary cancer metastasis in the central lymph nodes. There was also FTC in the right lobe of the thyroid and follicular carcinoma metastasis in the thoracic vertebra. In this case, the left lobe PTC had the BRAFV600E mutation, but there was no NRAS-Q61R mutation. The right lobe FTC and follicular cancer metastasised to the thoracic spine and had the NRAS-Q61R mutation, but there was no BRAF ${ }^{\mathrm{V} 600 \mathrm{E}}$ mutation.

In this case, for the diagnosis and treatment of the disease, the main point is that FNAC and FNA-Tg should be used before surgery, combined with molecular genetic testing to assess lymph nodes and determine the source and nature of the tumour, and select a suitable surgical method to ensure the curative effect while minimising surgical complications. The research results of Lee et al. [7] showed that the overall diagnostic accuracy of FNA for thyroid cancer is about $90 \%$, while the diagnostic rate for FTC is less than $60 \%$. For FTC, especially for micro-infiltrating FTC, FNAC is controversial. Because the diagnosis of FTC is based on whether the tumour has capsule invasion, vascular invasion, and extrathyroid metastasis, this is a phenomenon in the category of tissue structure. The cytological characteristics of FTC are not obvious in clinical practice, and it is difficult for FNAC to establish an accurate diagnosis of FTC [8]. Furthermore, it is also difficult to distinguish between follicular thyroid adenoma and FTC on intraoperative frozen sections [9, 10]. Generally, it is necessary to wait for a paraffin section to confirm the diagnosis of FTC, and, for micro-invasive FTC, if FNAC is performed before surgery, whether the tumour capsule will be damaged and affect the diagnosis of FTC. Therefore, for simple follicular tumours, preoperative FNAC is controversial. However, the guidelines for the diagnosis and treatment of adult thyroid nodules and differentiated thyroid cancer (American Thyroid Association 2015) indicate that atypical follicular lesions and follicular tumour nodules with RAS mutation are $84 \%$ more likely to be malignant lesions. The risk of malignant lesions in suspicious nodules with BRAFV600E mutation is > 95\% [11], so FNAC can be combined with genetic testing such as RAS and BRAF ${ }^{\mathrm{V} 600 \mathrm{E}}$ to help the diagnosis of this disease. In this case, the postoperative pathology is PTC combined with extensive invasive FTC, and the source and nature of tumours invading subcutaneous tissue to the epidermis were unknown before the operation. If FNAC is beneficial before the operation, it is conducive to the diagnosis of PTC. It is also helpful to have a better understanding of the source and nature of tumours that invade the epidermis. When molecular tests of BRAF ${ }^{\mathrm{V} 600 \mathrm{E}}$ and RAS genes are available, the source and nature of tumours that invade the subcutaneous tissue to the epidermis can be better judged. For the assessment of lymph nodes, in this case, the nature of the lymph nodes in the right cervical area IV of the neck colour Doppler ultrasound needed to be determined and tended to be malignant, but postoperative pathology confirmed that there was no lymph node metastasis. Analysis of the corresponding literature shows that although metastatic lymph nodes often have volume and morphological changes, the disappearance of lymph hilus structure, hyperechoic masses, cystic degeneration, and calcification on ultrasound [12] means that colour Doppler ultrasound is only an auxiliary examination and cannot be used as a standard for the diagnosis of lymph node metastasis of thyroid cancer. To 
further improve the diagnostic accuracy of thyroid lymph node metastasis, FNAC can be used to confirm the diagnosis. Generally speaking, excluding ectopic thyroid and observing thyroid follicular cells in lymph nodes can diagnose lymph node metastasis of thyroid cancer, or the thyroglobulin (Tg) in eluate from fine needle aspiration (FNA-Tg) can also be measured during the FNAC process to improve the accuracy of the diagnosis further [13]. The guidelines indicate that FNA cytology can be performed on suspicious lymph nodes with a small diameter of 8-10 mm, and the level of Tg in the washing fluid can be measured. Tg concentration $\geq 1 \mu \mathrm{g} / \mathrm{l}$ is suspicious for metastasis. The higher the Tg level, the greater the possibility that the lymph nodes are metastatic, and the result is not affected by thyroglobulin antibodies [11]. In this case, because the source and nature of the anterior neck tumour were not clearly identified before the operation, the evaluation of the nature of the lateral neck lymph nodes was not clear, and the patient and family members had a strong willingness to operate. They expressed their refusal to puncture and hoped that preventive dissection of the contralateral cervical lymph nodes could be performed. The intraoperative findings and frozen pathology suggested that the tumour was very aggressive. Therefore, bilateral cervical lymph node dissection was performed, but no lateral cervical lymph node metastasis was found after the operation. In summary, reflecting on the treatment process, FNAC and FNA-Tg should be used before surgery, combined with molecular genetic testing to assess lymph nodes and determine the source and nature of the tumour, choose a suitable surgical method, and ensure the curative effect while minimising surgical complications.

There is no large-scale study explaining the difference between the prognosis of the disease and the general prognosis of PTC or FTC, but some literature [14] reports that the prognosis of patients with medullary thyroid cancer (MTC) combined with PTC is no different from that of patients with general MTC. The co-occurrence of different types of thyroid cancer will not have a significant impact on the prognosis of a single type of thyroid cancer. In this case, after active surgery, during the 4-year follow-up of the patient, the endocrine suppression treatment was satisfactory, and the ultrasound examination of the neck showed no abnormalities. There were no signs of recurrence or metastasis.

In conclusion, although medical records of PTC combined with FTC are rare, the use of auxiliary examinations such as FNAC, FNA-Tg, and genetic molecular detection methods can provide patients with more standardised treatment, early detection, early diagnosis, and active surgery, which can improve the prognosis.

\section{Acknowledgments}

The first author is Wei Yan.

Fund name: Application of ultrasonography in the diagnosis of hashimoto's thyroiditis complicated with micropapillary carcinoma.

Project number: Department of Science and Technology of Guizhou Province and Affiliated Hospital of Guizhou Medical University Contract No. [2015]7412.

\section{Conflict of interest}

The authors declare no conflict of interest.

\section{References}

1. Davies L, Welch HG. Increasing incidence of thyroid cancer in the United States, 1973-2002. JAMA 2006; 295: 2164-7.

2. Tiejun L. On the pathogenesis, diagnosis and treatment of thyroid cancer. Chin Health Industry 2014; 27: 137-8.

3. Schlumberger M, Sherman SI. Approach to the patient with advanced differentiated thyroid cancer. Eur J Endocrinol 2012; 166: 5-11.

4. Hsiao SJ, Nikiforov YE. Molecular approaches to thyroid cancer diagnosis. Endocr Relat Cancer 2014; 21: T301.

5. Pastolero GC, Coire Cl, Asa S L. Concurrent medullary and papillary carcinomas of thyroid with lymph node metastases. A collision phenomenon. Am I Surg Pathol 1996; 20: 245-50.

6. Cracolici V, Mujacic I, Kadri S, et al. Synchronous and metastatic papillary and follicular thyroid carcinomas with unique molecular signatures. Am J Surg Pathol 2017; 29: 1-6.

7. Lee TI, Yang HJ, Lin SY, et al. The accuracy of fine-needle aspiration biopsy and frozen section in patients with thyroid cancer. Thyroid 2002; 12: 619-26.

8. Somma J, Schlecht NF, Fink D, et al. Thyroid fine needle aspiration cytology: follicular lesions and the gray zone. Acta Cytol 2010; 54: 123-31.

9. Sillery JC, Reading CC, Charboneau JW, et al. Thyroid follicular carcinoma: sonographic features of 50 cases. Am J Roentgenol 2010; 194: 44-54.

10. Lai XJ, Zhang B, Jiang YX, et al. Usefulness of ultrasonography in the differential diagnosis of thyroidal follicular tumor. Zhongguo Yi Xue Ke Xue Yuan Xue Bao 2013; 35: 483-7.

11. Zhang B, Xu JZ, Wu Q, et al. Interpretation of the 2015 American Thyroid Association "Guidelines for the diagnosis and treatment of adult thyroid nodules and differentiated thyroid cancer": ultrasound section. Chin J Cancer 2016; 26: 19-24.

12. Liu Z, Zeng W, Liu C, et al. Diagnostic accuracy of ultrasonographic features for lymph node metastasis in papillary thyroid microcarcinoma: a single-center retrospective study. World I Surg Oncol 2017; 15: 32.

13. Zhou L, Zhang G, Zhang DQ, et al. Application research of fine needle aspiration biopsy and eluate detection in decision-making of cervical lymph node dissection for thyroid cancer. Chin J Practical Surg 2017; 37: 1002-6.

14. Wang B, Jiang K, Lei JY, et al. Analysis of clinical characteristics of medullary thyroid carcinoma combined with papillary carcinoma. Chin J Practical Surg 2017; 037(003): 299-302. 